Iran Journal of Nursing (IJN)

Vol 34, No. 132, Oct 2021: 8- 20

\title{
Evaluation of the Relationship between Life Quality and Circadian Types and Anxiety in Iranian Epilepsy Association Members with Epilepsy in
} 2020

\author{
Mahnaz Seyedoshohadaee ${ }^{1}$, Ghazaleh Salighedar², Hamid Haghani ${ }^{3}$
}

\begin{abstract}
Background \& Aims: Epilepsy is one of the most common chronic neurological disorders, and epileptic patients are prone to physical and psychological diseases and can experience issues such as anxiety, which affect their life quality. In general, epilepsy reduces life quality indexes and life expectancy, which has considerable economic effects on the person and the society and will ultimately lead to problems such as isolation, dependence, psychological damages and disorders, remaining single, unemployment and declining quality of life. Daily preferences and circadian rhythms are recognized as factors affecting epileptic patients' life quality. As a mental feeling, quality of life is a psychological phenomenon that may play a role in patients' life quality through personal traits such as daily preferences and circadian rhythms. In other words, circadian rhythms and anxiety are important issues found in epileptic patients, which can affect their quality of life. Therefore, the present study aimed to determine the relationship between life quality and circadian rhythms and anxiety in epileptic patient members of the Iranian Epilepsy Association.

Materials \& Methods: This was a cross-sectional, descriptive and correlational study performed on 120 individuals who referred to the Iranian Epilepsy Association. The participants were selected by continuous sampling and based on the inclusion criteria. The sampling process continued for a period of June-August, 2020. Data were collected using a demographic characteristics questionnaire, including age, gender, level of education, marital status, occupational status, average monthly family income, and medical information related to other diseases, duration of diagnosis, and diagnosis of epilepsy in other family members. In addition, we applied the Morningness-Eveningness Questionnaire (MEQ), which comprises 19 questions about the sleep and waking times and appropriate times for physical, mental and conscious functioning after waking up. Moreover, we used the Beck Anxiety Inventory (BAI), which has 21 items about cognitive and physical signs of anxiety. Furthermore, we exploited the Quality of Life in Epilepsy (QOLIE-31-P) was exploited, which encompasses subscales of concern about epilepsy, psychological health, energy/fatigue, cognitive performance, drug effects, social performance and overall quality of life. The instruments were filled by the participants in a predetermined location through self-report. Notably, the process took 20-30 minutes per subject. Data analysis was performed in SPSS version 16 using descriptive, inferential and correlational statistics and multiple linear regression model to determine the predictors of quality of life in patients with epilepsy.

Results: In this study, the mean age of the subjects was 4.14 years. Regarding circadian rhythms, most participants were in an intermediate position $(71.7 \%)$, which had the highest frequency compared to other circadian rhythms. However, the lowest frequency was related to the complete morningness state. On the other hand, there was no significant relationship between life quality scales and circadian rhythms. Quality of life had the highest score in the dimension of seizure anxiety with an average of 57.47 and in the dimension of social
\end{abstract}

\footnotetext{
1. Department of Internal- Surgical Nursing, School of Nursing and Midwifery, Iran University of Medical Sciences, Tehran, Iran

2. Department of Internal- Surgical Nursing, School of Nursing and Midwifery, Iran University of Medical Sciences, Tehran, Iran (Corresponding Author) Tel: $02143651000 \quad$ Email: salighehdar.gh@iums.ac.ir

3. Department of Biostatistics, School of Health, Iran University of Medical Sciences, Tehran, Iran
} 
functioning with an average of 45.76 had the lowest mean score among other dimensions. Moreover, the average score of quality of life was 50.32 with a standard deviation of 23.03 , which was close to the median of the instrument score- i.e., 50. There was a significant negative correlation between the quality of life and all of its dimensions with anxiety, meaning that quality of life and its dimensions decreased with an increase in anxiety $(\mathrm{r}=-0.673, \mathrm{P}<-0.001)$. In addition, a significant association was observed between anxiety and variables of the level of education, occupational status, income level and hospitalization frequency. Moreover, diagnosis of other diseases $(\mathrm{P}=0.001)$ and five-six hospitalization times $(\mathrm{P}<0.001)$ were the only significant variables in the regression model.

Conclusion: According to the results of the study, there was a negative significant correlation between the quality of life and all of its dimensions with anxiety. It seems that effective steps could be taken toward promoting anxiety-free behaviors, which increases the life quality of patients with epilepsy, through raising awareness, considering a minimum level of education of high school diploma as a recruitment criterion, creating jobs with suitable income levels, and eliminating organizational barriers (e.g., facilitation of staff recruitment).

\section{Keywords: Epilepsy, Anxiety, Quality of Life, Circadian types}

\section{Conflict of Interest: No}

How to Cite: Seyed Shohadaee M, Salighedar GH, Haghani H. Evaluation of the Relationship between Life Quality and Circadian Types and Anxiety in Iranian Epilepsy Association Members with Epilepsy in 2020. Iran Journal of Nursing. 2021; 34(132):8-20.

Received: 5 Jul 2021

Accepted: 4 Oct 2021 


\title{
ارتباط كيفيت زندكى با تيّهاى شبانه روزى و اضطراب افر اد با اختلال صرع عضو انجمن

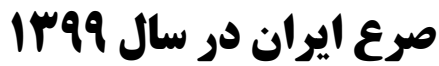

\author{
مهناز سيدالشههدايى'، غزاله سليقه دارّ، حميد حقانى
}

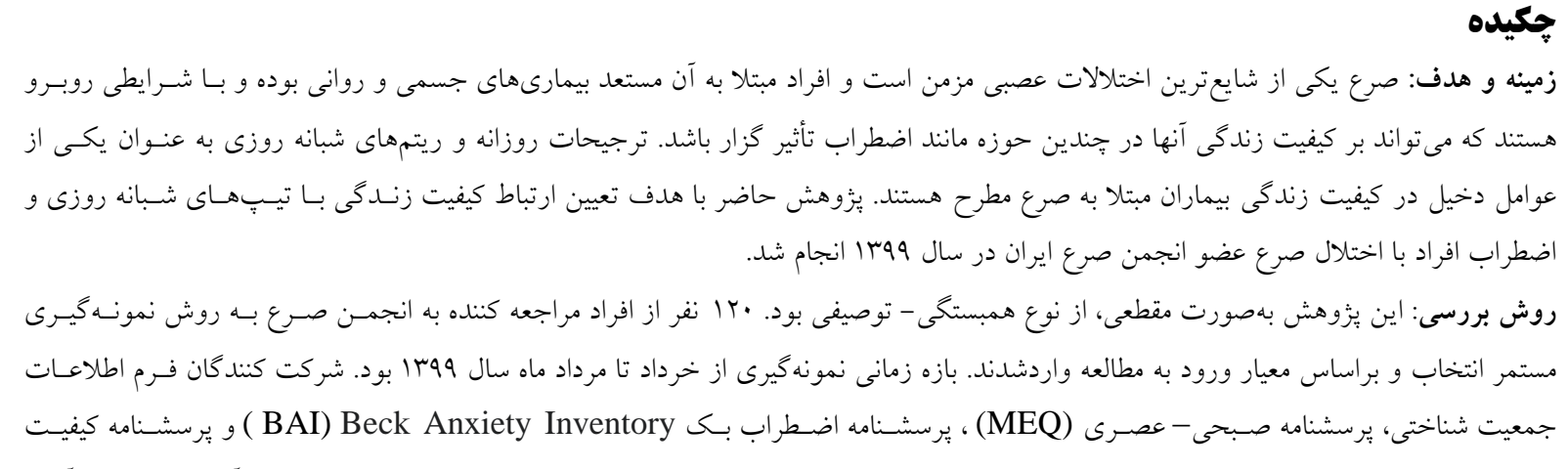

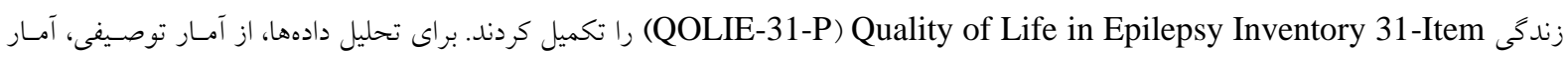

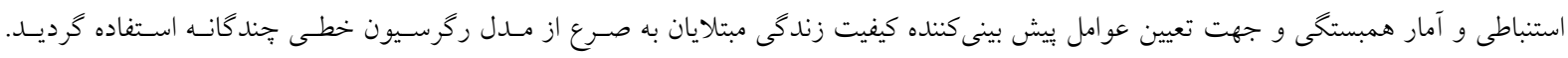

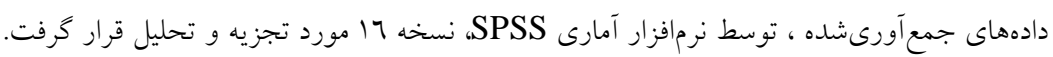

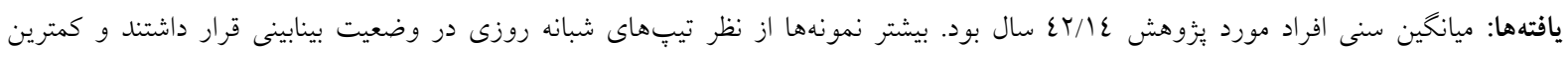

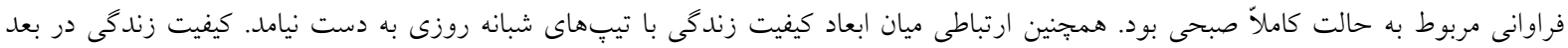

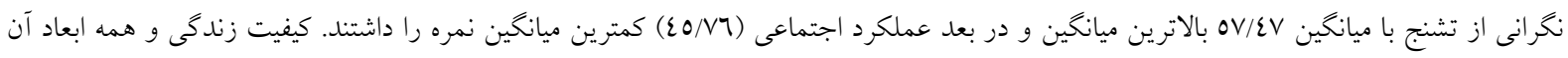

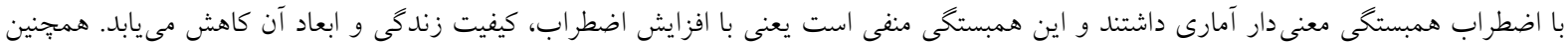

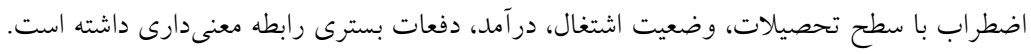

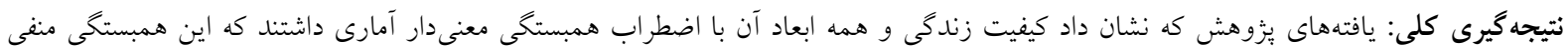

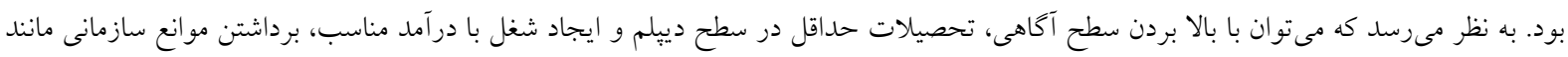

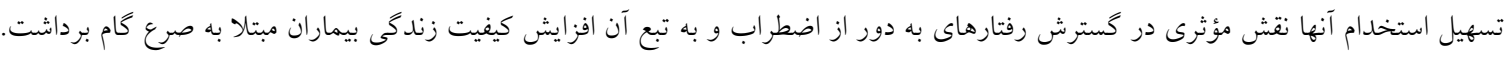

$$
\text { تارضيد وازهها: صرع، اضطراب، كيفيت زندگى، تيبهاى شبانه روزى }
$$

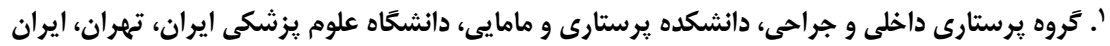

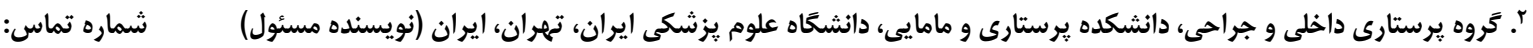


صبح بالاتر است. در مقابل عصرىها هنخـام شـب بيـدار

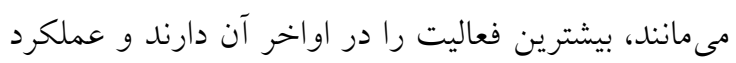

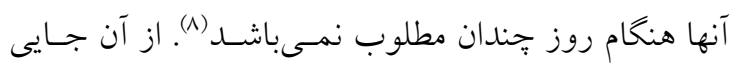

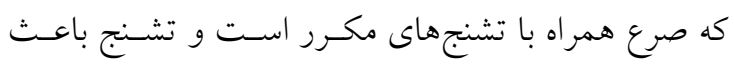

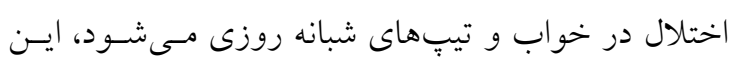

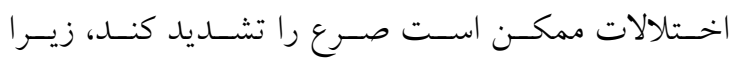

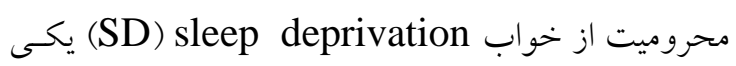

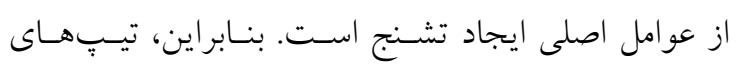

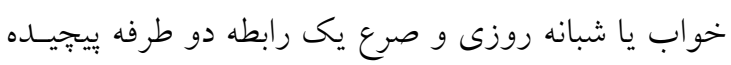

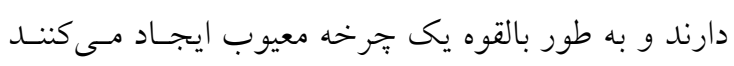

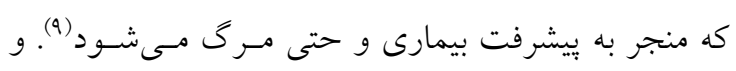

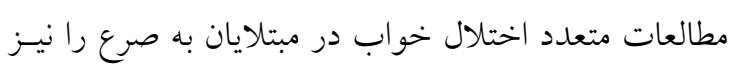
تأييد كردهاند (11،.1). مطالعات نشان دادهاند كه بيماران مبتلا به صرع به شدت

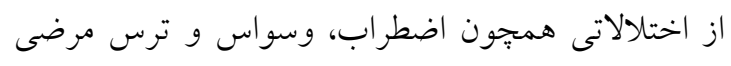

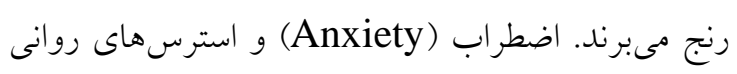
از عوامل افزايش دهنده خطر خودكشى در بيماران مبتلا

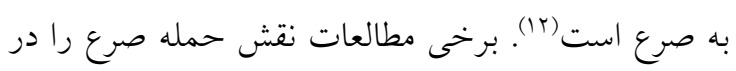

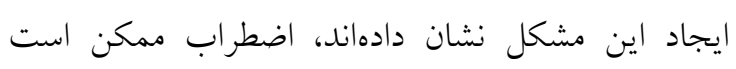

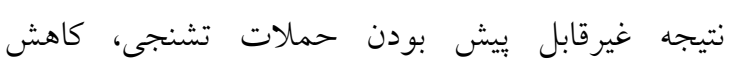

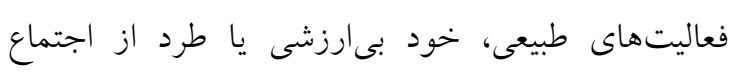

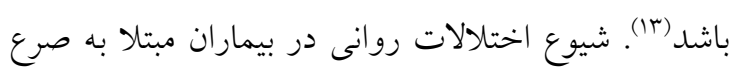

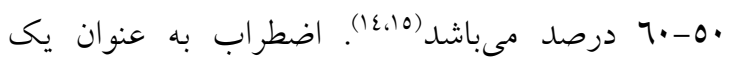

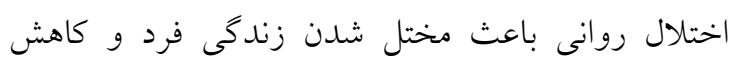

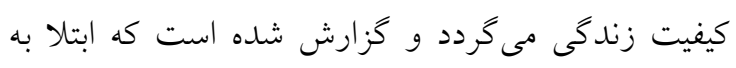

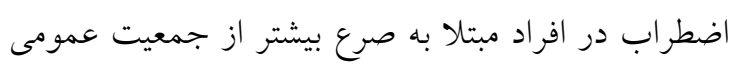
است (17) به طورى كه اضطراب مىتواند به طور مستقيم

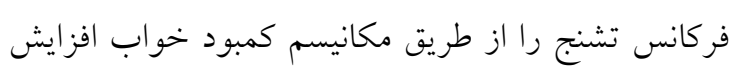

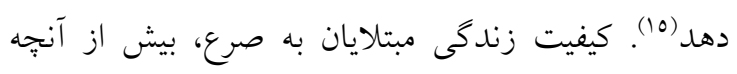

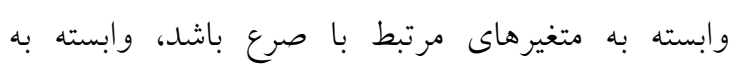

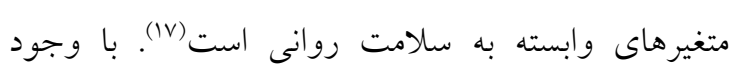

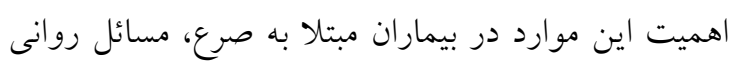
آنان كمتر مورد تشخيص و درمان قرار كرفته است (1).
صرع يا Epilepsy به عنوان يكى از اخــتلالات شــايع

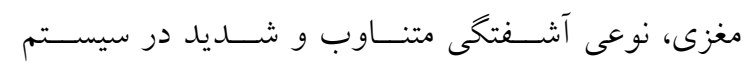

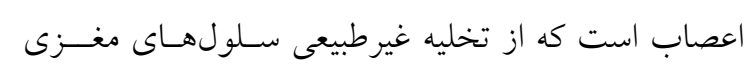

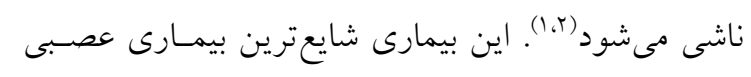

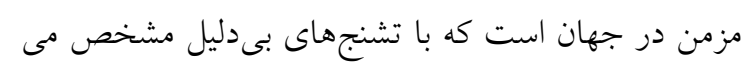

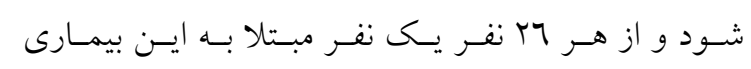

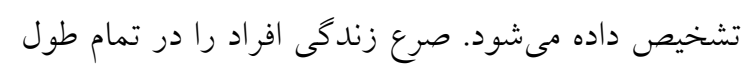

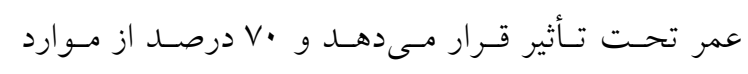

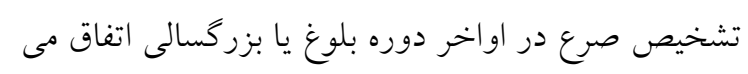

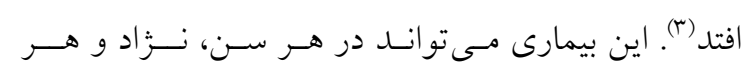

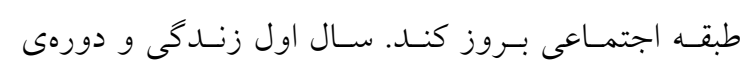

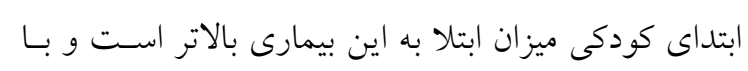

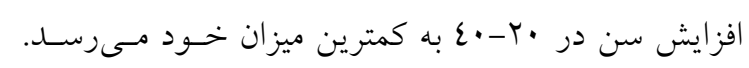

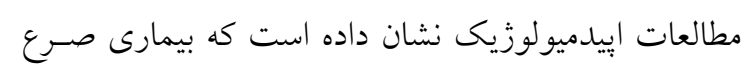
مى تو اند شرايط فرهنكى، مـذهبى، آموزشسى، اجتمـاعى و اقتصادى را تحت تأثير قرار دهد (ع).

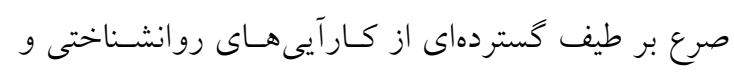

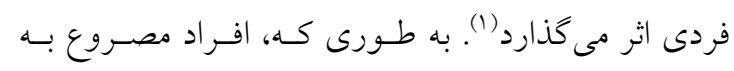

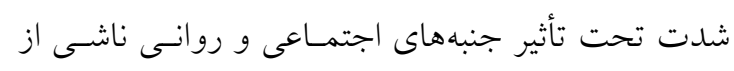

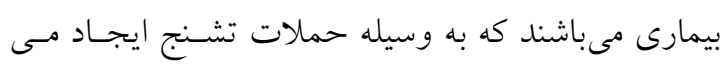

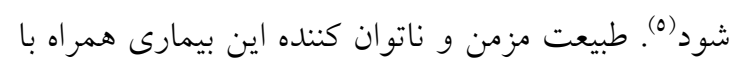

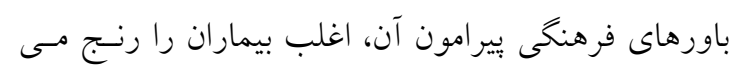

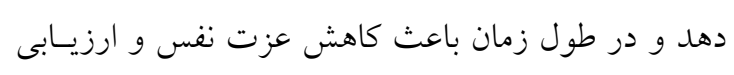

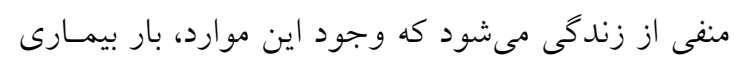

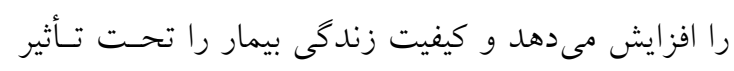

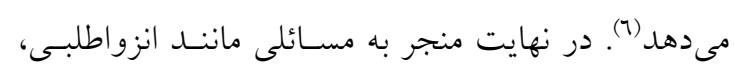

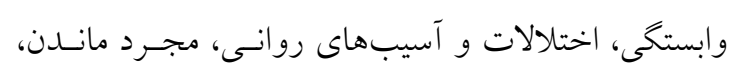

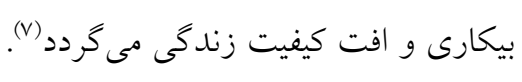

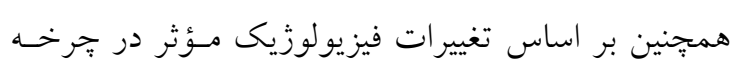

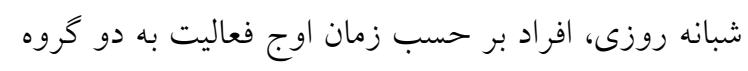

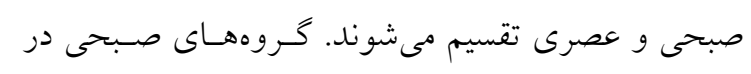

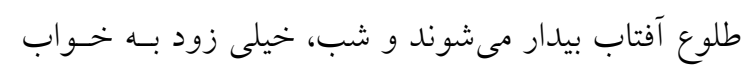

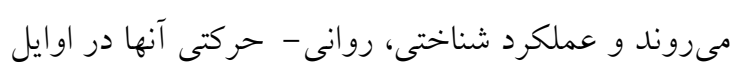


نظركرفته شد. و با فرض اينكه ضريب همبستكى كيفيـت

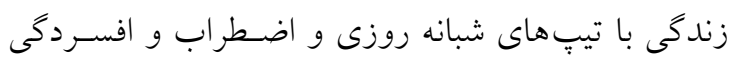

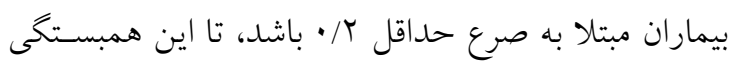

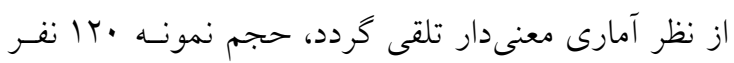
در هر گروه برآورد گرديد.

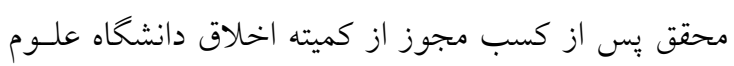

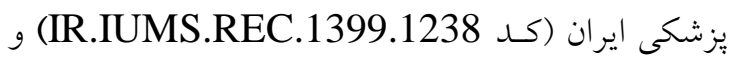
دريافت معرفى نامه از دانشكده يرستارى و مامايى جهـت نمونه گيرى به روش در دسترس به انجمن صسرع مراجعسه نمود. ابزار مورد استفاده در اين يزوهش شامل فرم () اطلاعات جمعيت شناختى (سن، جنس، ميزان تحصيلات، وضعيت

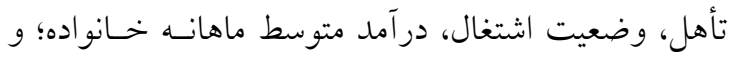
اطلاعات طبى شامل ابتلا به بيمارىهاى ديخر، مدت زمات زمان

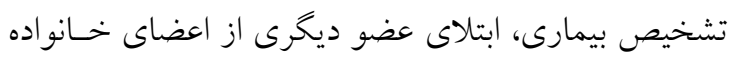
به بيمارى صرع)، Y) يرسشنامه صبحى - عصرى (MEQ) Morningness-Eveningness Questionnaire كه توسط Horne و Stenberg طراحى شده بـود. ايسن يرسشنامه يك ابزار خود كزارش دهى اسـت و شـامل 19 سؤال است كه مربوط به زمـان خو ابيــن و بيــار شــدن، زمـانهـاى ترجيحسى بــراى عملكــــ فيزيكس، ذهنسى و هوشيارى بعد از بيدارى است. باسخ ها در مقياس ليكـرت

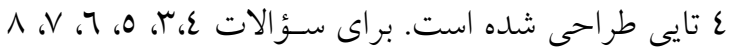

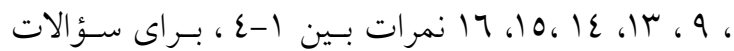

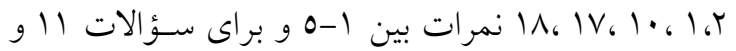

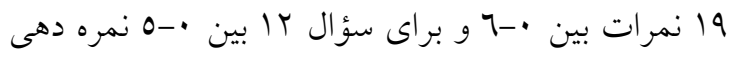

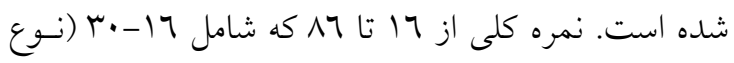

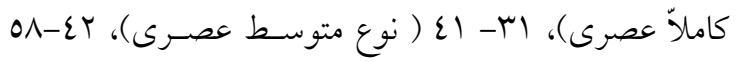

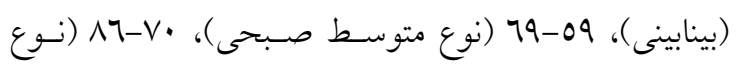

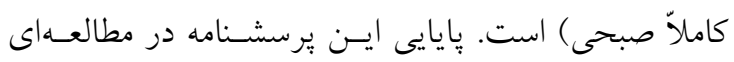

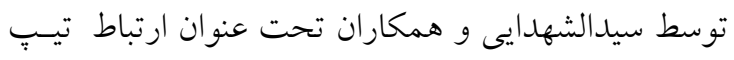

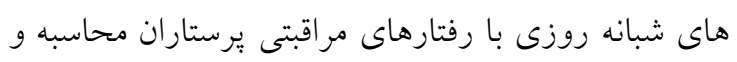

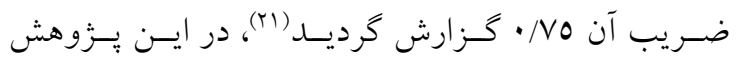

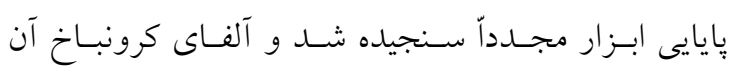

مطالعات اندكى در ايران بر روى فراوانى اختلالات روانى

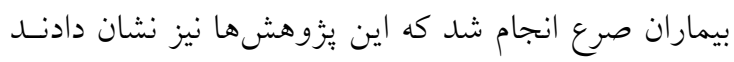

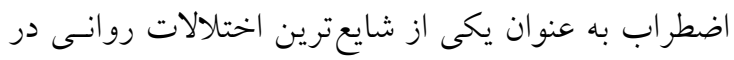

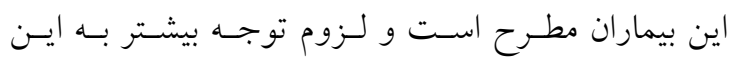

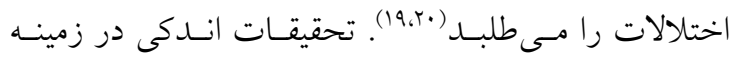

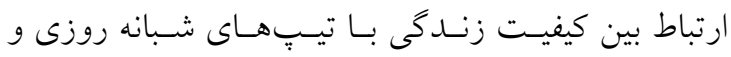

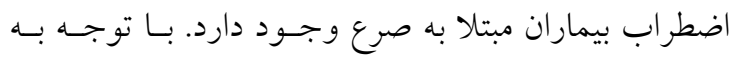

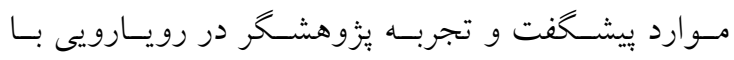

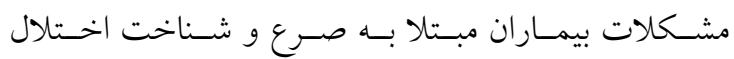
اضطراب و ريتمهاى شبانه روزى در بيماران مبتلا و تـأثير آن بر كيفيت زندكى در جهت بهينه سازى مديريت تشنج،

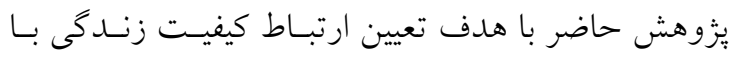

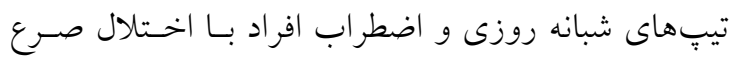
عضو انجمن صرع ايران انجام گرديد.

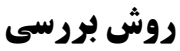

اين مطالعه مقطعى از نوع همبستخى توصيفى بـود كـه در

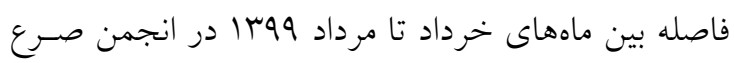

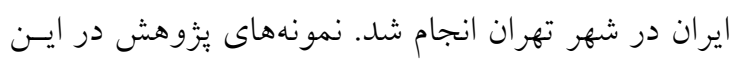

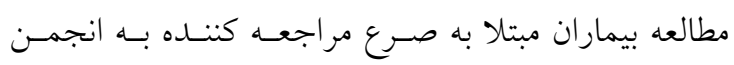

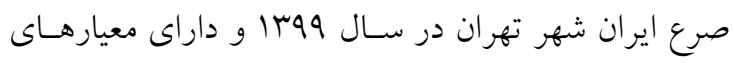

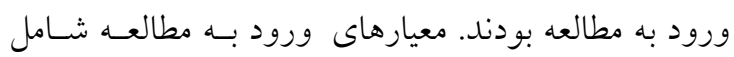

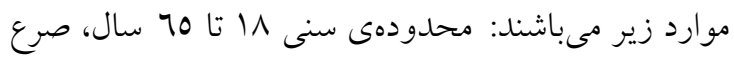

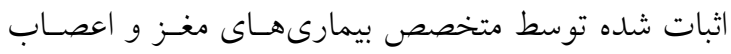

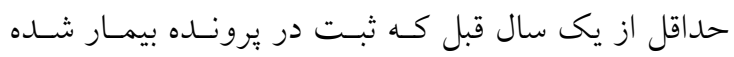
باشد، EEG ثبت در يرونده، انجام نشــدن مشـاوره روان

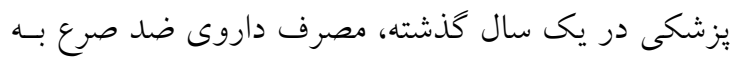

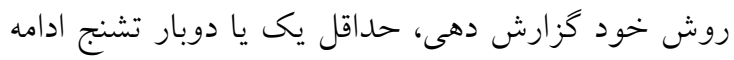
دار در سال كذشته، توانـايى خوانسـن و نوشـتن بـه زبــان فارسى و توانايى ياسخكويى به سـؤالات يرسشــامههـا از

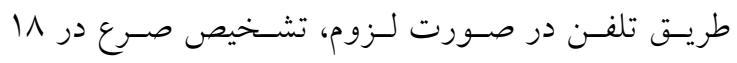

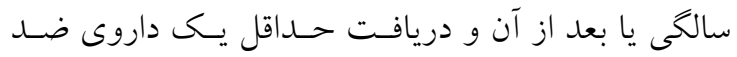

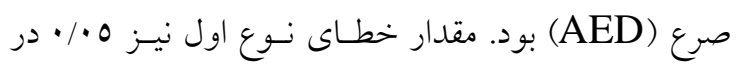

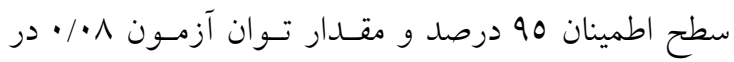




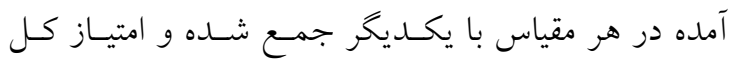

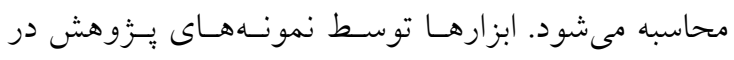

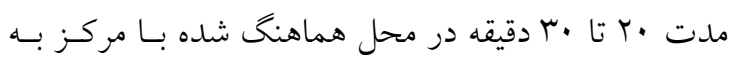

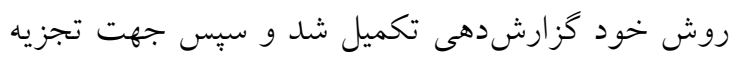

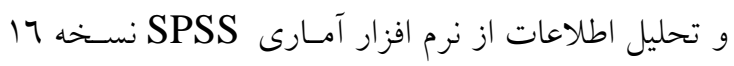

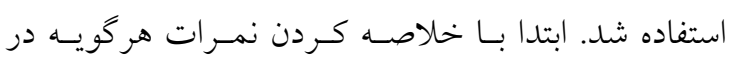

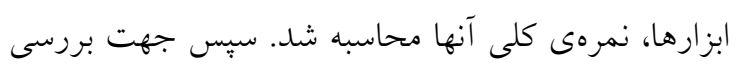

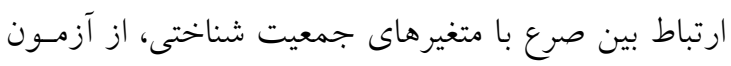

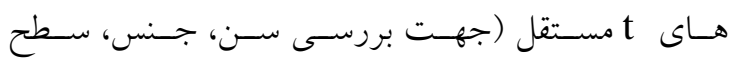
تحصيلات، وضعيت تأهل، وضعيت اشتغال، كفايت درآمد

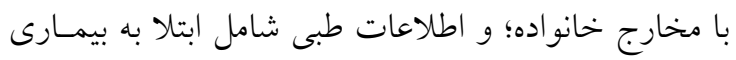

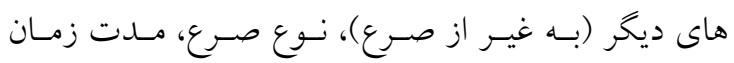
تشخيص بيمارى، ابتلاى عضو ديخرى از اعضاى خـانو اده

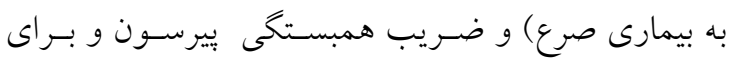

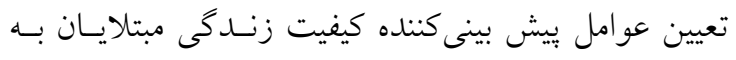

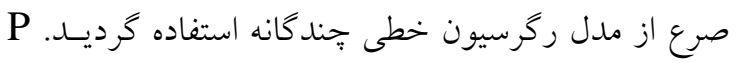

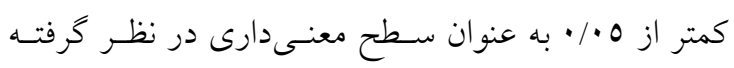

\section{يافتهها}

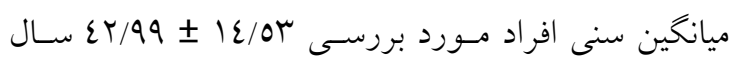

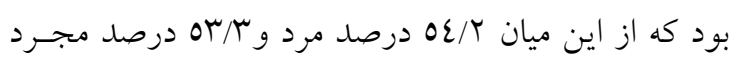

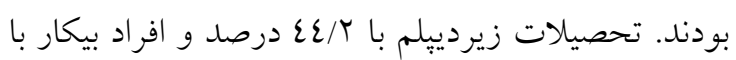

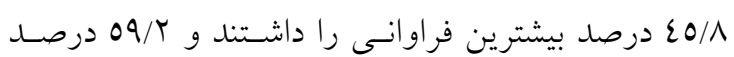

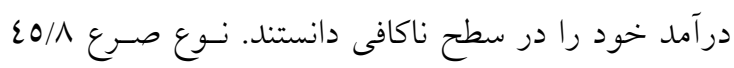

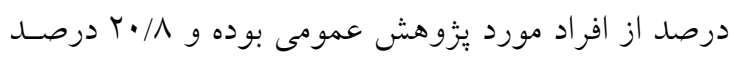

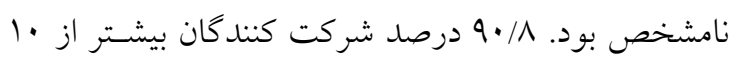

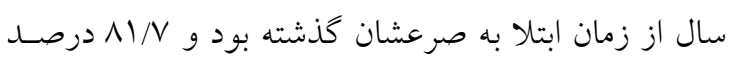

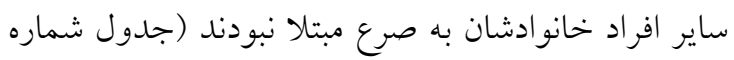

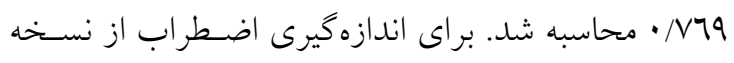

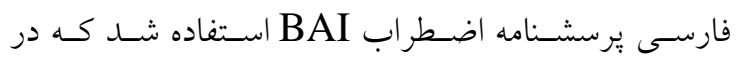
مطالعات يِيشين روايى و بِايايى آن مورد تأييد قرار كرفتسه.

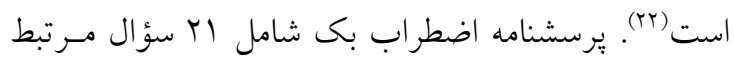

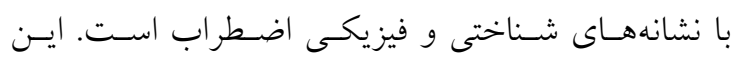

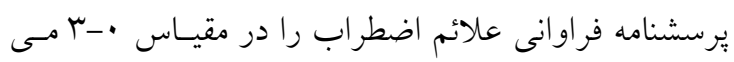

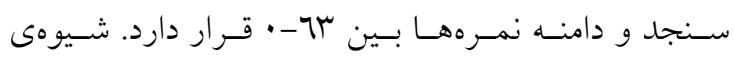

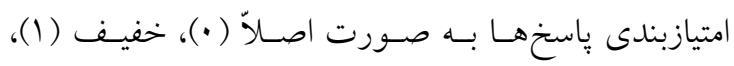

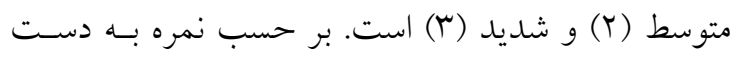

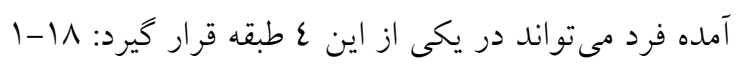

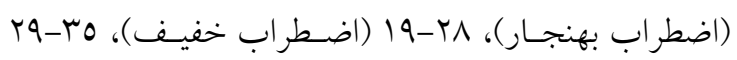

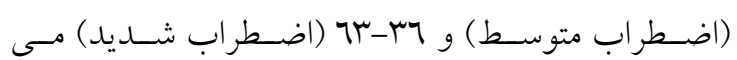

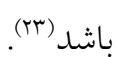

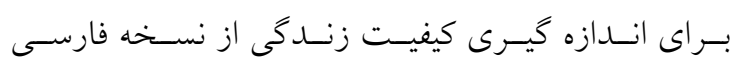

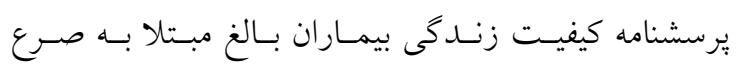
(QOLIE-31-P) Quality of Life in Epilepsy استفاده شد كه در مطالعهى متتظرى و همكـاران روايسى و

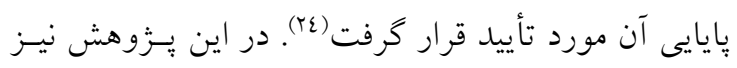

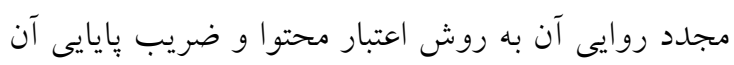

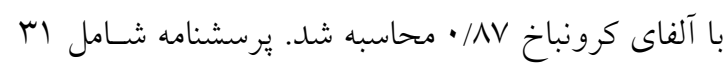

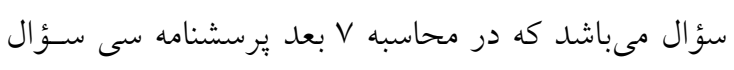

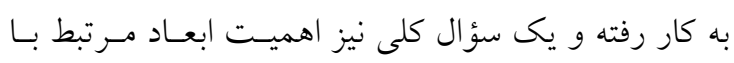
بيمارى را از نظر بيمار بررسى مى نمايد. ابعاد ابزار عبارتند

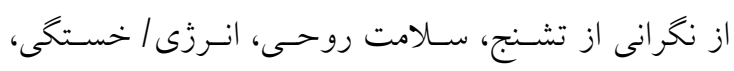

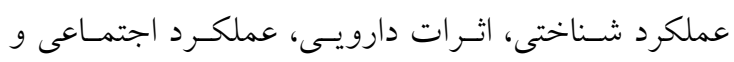

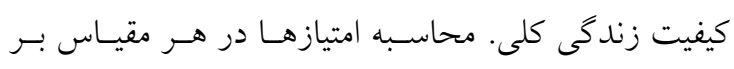

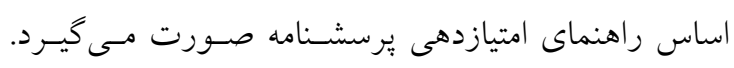
محدوده امتيازها در هر مقيـاس از ·-..' بــوده و امتيـاز

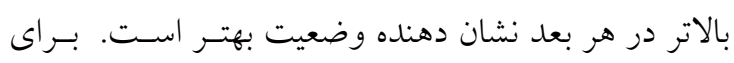

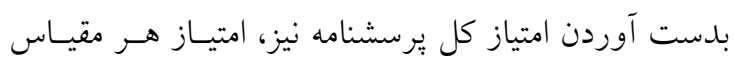

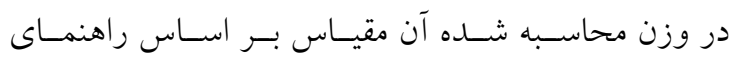

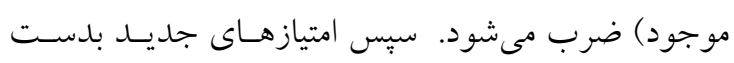


جدول شماره (: توزيع فراوانى مشخصات فردى و بيمارى افراد با اختلال صرع مراجعه كننده به انجمن صرع ايران- سال 99"

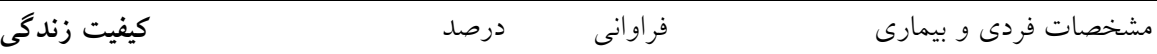

\begin{tabular}{|c|c|c|c|c|c|}
\hline نتيجه آزمون & انحراف معيار 土 ميانخين & & & & \\
\hline $\mathrm{t}=\cdot / \mathrm{N} r \mathrm{r} \quad \mathrm{df}=111$ & $01 / V \varepsilon \pm r r / q$ & $0 \varepsilon / r$ & 70 & مرد & جنسيت \\
\hline $\mathrm{P}=\cdot / \varepsilon \tau \pi$ & $\varepsilon \Lambda / T r \pm r \mu / r \Lambda$ & $\varepsilon 0 / \wedge$ & 00 & 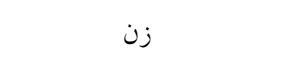 & \\
\hline $\mathrm{t}=\cdot 10 \mathrm{\vee} 9 \mathrm{df}=1111$ & $01 / \mu \pi \pm r r / \Lambda$ & or/ & $7 \varepsilon$ & مجرد & وضعيت تأهل \\
\hline $\mathrm{P}=\cdot / 07 \varepsilon$ & 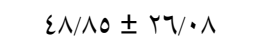 & $\varepsilon \cdot / \wedge$ & $\varepsilon 9$ & 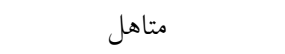 & \\
\hline $\mathrm{F}=\Lambda / r \Delta \wedge$ & $\varepsilon \varepsilon / 90 \pm r \cdot / 0$ & $\varepsilon \varepsilon / r$ & or & زيردييلم & \\
\hline $\mathrm{P}<\bullet / \bullet \cdot 1$ & $\varepsilon 0 / 9 r \pm r r / 0 \Lambda$ & YA/r & $r \varepsilon$ & دييلم & سطح تحصيلات \\
\hline & $T r / \varepsilon V \pm r r / N r$ & TV/O & 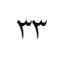 & دانشخاهى & \\
\hline $\mathrm{F}=0 / \cdots \varepsilon$ & $V \cdot / K \varepsilon \pm 1 / / 70$ & 1. & ir & كارمند & \\
\hline $\mathrm{P}=\cdot / \cdot \cdot 1$ & $T / / r 0 \pm r \varepsilon / 1 \varepsilon$ & 10 & $1 \wedge$ & آزاد & وضعيت اشتغال \\
\hline & $\varepsilon \varepsilon / 7 \pm r r / \wedge 0$ & $Y I / N$ & $r 7$ & خانه دار & \\
\hline & $\sum \tau / V T \pm Y \cdot / 71$ & $\varepsilon 0 / \wedge$ & 00 & بيكار & \\
\hline & $\varepsilon \cdot / \varepsilon \cdot \pm \mid \Lambda / \Lambda \Lambda$ & $V / 0$ & 9 & بازنشسته & \\
\hline $\mathrm{t}=\varepsilon / \cdot \varepsilon \mathrm{V} \quad \mathrm{df}=11 \wedge$ & $09 / 97 \pm r 1 / 97$ & $\varepsilon \cdot / \wedge$ & $\varepsilon 9$ & كافى & كفايت درآمد با \\
\hline $\mathrm{P}<\bullet / \cdot \cdot 1$ & $\varepsilon r / T V \pm r \mid / \varepsilon V$ & $09 / r$ & VI & 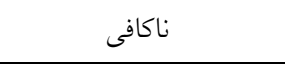 & مخارج خانواده \\
\hline$t=r / 011 \quad d f=111$ & $\{r / 99 \pm r r / r\}$ & $\varepsilon$. & $\varepsilon \wedge$ & بله & ابتلا به بيمارى ديخر \\
\hline $\mathrm{P}=\cdot / \cdot 1 r$ & $O \varepsilon / O \varepsilon \pm r r / V O$ & 7. & Vr & خير ير & \\
\hline $\mathrm{F}=\cdot / \mathrm{\varepsilon} r$ & $\varepsilon \Lambda / \mu^{\prime} \pm \pm r / r_{0}$ & $\varepsilon 0 / \wedge$ & 00 & عمومى ع ل ع & \\
\hline $\mathrm{P}=\cdot / \varepsilon \vee \wedge$ & $0 r / 90 \pm r r / 20$ & 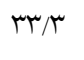 & $\varepsilon$. & كانونى & نوع صرع \\
\hline & $\sum \Lambda / V O \pm$ TV/OQ & $r \cdot / \Lambda$ & ro & نامشخص & \\
\hline $\mathrm{t}=\cdot / \mu \mathrm{r} \mid \mathrm{df}=11 \wedge$ & $O T / \Sigma O \pm Y r / \Sigma O$ & $9 / 4$ & 11 & كمتر از ده سال & مدت زمان تشخيص \\
\hline $\mathrm{P}=\cdot / \vee \varepsilon \wedge$ & $0 . / 11 \pm r r / 77$ & $9 \cdot / 1$ & 1.9 & بيشتر از ده سال & صرع \\
\hline $\mathrm{t}=\cdot 1077 \mathrm{df}=111 \mathrm{~A}$ & $O r / A \varepsilon \pm$ YO/乏O & $1 / / \pi$ & rt & بله & ابتلاى ساير افراد \\
\hline $\mathrm{P}=\cdot / 0 \mathrm{VT}$ & $\sum 9 / V 0 \pm r r / 00$ & $11 / \mathrm{V}$ & 91 & خير & خانو اده به صرع \\
\hline$r=-\cdot / 177$ & & \multicolumn{2}{|c|}{$\varepsilon r / 99 \pm 1 \varepsilon / 0 r$} & انحراف معيار 土 ميانخين & سن (سال) \\
\hline $\mathrm{P}=\cdot / \cdot \mathrm{V}$ & & \multicolumn{2}{|c|}{$M \Lambda-V Y$} & بيشينه - كمينه & \\
\hline
\end{tabular}

ميانخين نمره را در بين ساير ابعاد داشتند و كيفيت زندكى

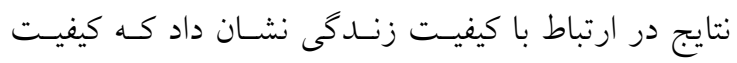

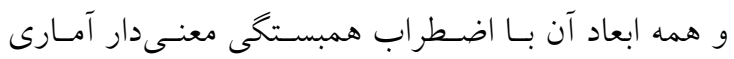

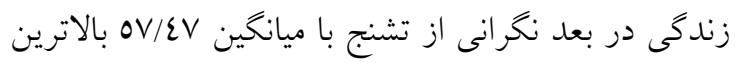

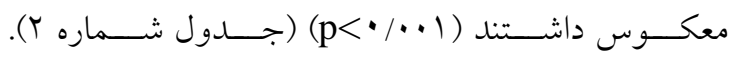

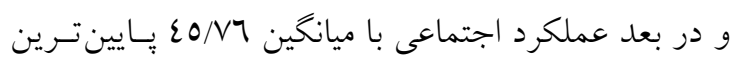

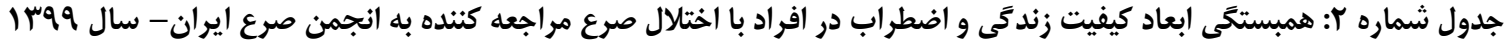

\begin{tabular}{|c|c|c|c|}
\hline \multicolumn{2}{|c|}{ اضطر اب } & انحراف معيار \ ميانگين & كيفيت زندگى و ابعاد آن \\
\hline $\mathrm{r}=-\cdot / 2 q r$ & $\mathrm{P}<\cdot / \cdot \cdot 1$ & $O V / \Sigma V \pm \Gamma N / Y r$ & نخرانى از تشنج \\
\hline $\mathrm{r}=-\cdot / 29 \mathrm{~V}$ & $\mathrm{P}<\cdot / \cdot \cdot 1$ & $0 \cdot / \Lambda T \pm r \cdot / \Lambda V$ & سلامت روحى \\
\hline $\mathrm{r}=-\cdot / \Gamma \wedge \mid$ & $\mathrm{P}<\cdot / \cdot \cdot 1$ & $\varepsilon V / 01 \pm 19 / 70$ & انرزى/ خستخى \\
\hline $\mathrm{r}=-\cdot \cdot 1070$ & $\mathrm{P}<\cdot / \cdot \cdot 1$ & $\varepsilon \tau / \varepsilon \pm r_{0} / r_{0}$ & عملكرد شناختى \\
\hline$r=-. / 077$ & $\mathrm{P}<\cdot / \cdot \cdot 1$ & $O V / T O \pm T_{0} / T_{q}$ & اثرات دارويى \\
\hline
\end{tabular}




\begin{tabular}{|c|c|c|c|}
\hline $\mathrm{r}=-\cdot / 29 \mathrm{~V}$ & $\mathrm{P}<\bullet / \cdot \cdot 1$ & $\varepsilon 0 / V\urcorner \pm r \varepsilon / r\urcorner$ & عملكرد اجتماعى \\
\hline $\mathrm{r}=-\cdot / 0 \mathrm{~V} 7$ & $\mathrm{P}<\cdot / \cdots 1$ & $\varepsilon T \cdot 0 \pm T r / V$ & كيفيت زندكى كلى \\
\hline $\mathrm{r}=-\cdot / \mathrm{TV}$ & $\mathrm{P}<\cdot / \cdots 1$ & $0 . / \mu T \pm r r / . r$ & نمره كل كيفيت زندگى \\
\hline
\end{tabular}

كيفيت زندگى و ابعاد آن ارتباط معنسى دار آمـارى نداشـته است (جدول شماره 0). بررسى ارتباط متغيرهاى فردى و

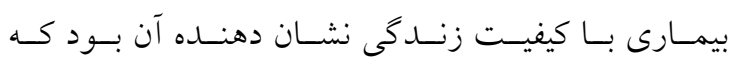

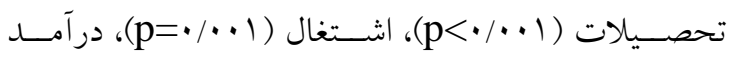

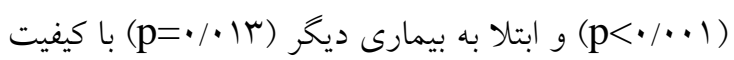
زندگى ارتباط معنى دار آمارى داشته است. مقايسـه دو بـهـ دو توكى نشان دهنده آن بود كه كيفيت زنـدكى در افـراد با تحصيلات دانشخاهى به طور معنسىدارى بـالاتر از زيـر

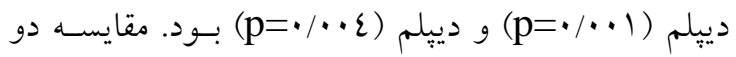
به دو در رابطه با وضعيت اشتغال نيز نشان دهنده آن بـود كـهـ كيفيـت زنــدكى در افـراد كارمنــــ بـالاتر از خانسهدار

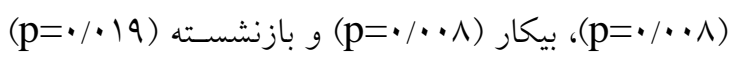
بود و در ساير سطوح اين اختلاف معنى دار نبود. همجنين نتايج نشان دهنده آن بود كه كيفيـت زنـدكى در افـراد بــا درآمد كافى و افرادى كه به بيمارى ديخرى مبـتلا نبودنــد،

$$
\text { بالاتر بود (جدول شماره ()). }
$$

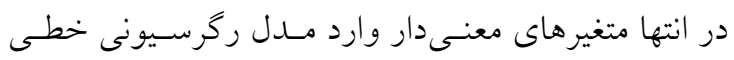

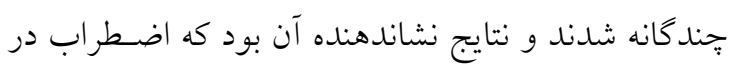

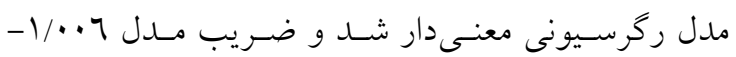

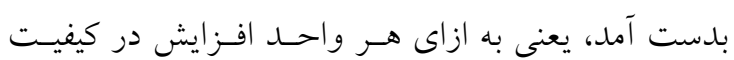

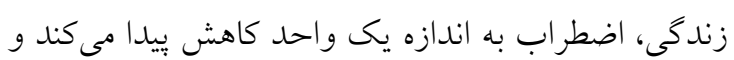
تحصيلات زيردييلم هم در مدل رگرسيونى معنى دار شــ

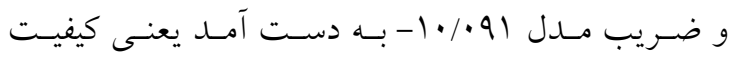
زنــدكى در افــراد بــا تحصـيلات زيـــدييلم نسـبت بــهـ دانشخاهى ·ل واحد كمتر بود (جدول شماره 7). لازم بـه ذكر است ضريب تعيين مدل OOH • به دست آمـد يعنى تنها 00 درصد از تغييرات متغير كيفيت زندكى تحت تأثير متغيرهاى وارد شده در مدل بودند.
يافته ها نشان داد كه فراوانسى اضـطراب بهنجـار بـا Tr/0

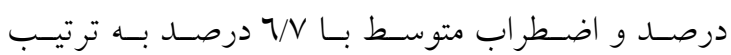
بيشترين و كمترين ميزان فراوانى را داشتند (جدول شماره (r)

جدول شماره سا: توزيع فراوانى اضطراب افراد با اختلال صرع

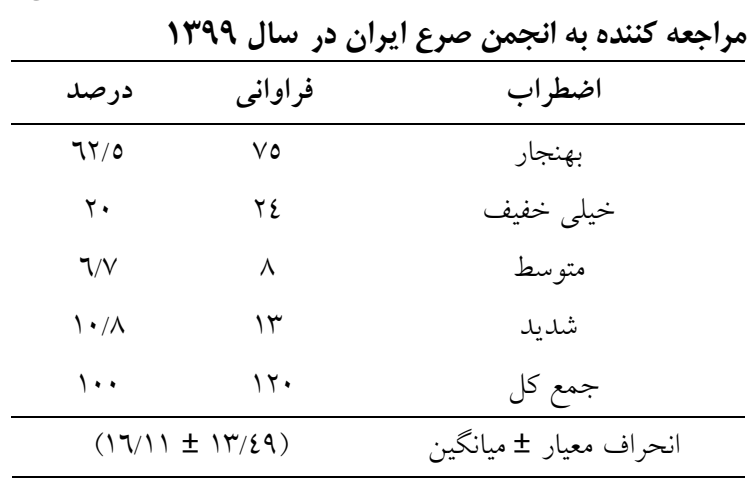

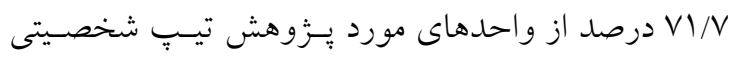

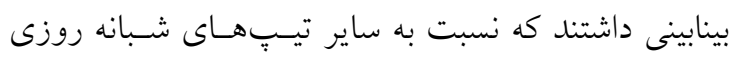
بيشترين فراوانى را داشت (جدول شماره ع).

جدول شماره ع: توزيع فراوانى تيبهای شبانه روزى افراد با

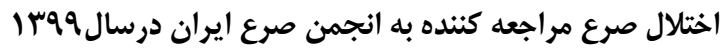

\begin{tabular}{|c|c|c|}
\hline درصد & فراوانى & تيبِهاى شبانه روزى \\
\hline$T / 0$ & r & عصرى متوسط \\
\hline$V I N$ & 17 & بينابينى \\
\hline$r \varepsilon / r$ & rq & صبحى متوسط \\
\hline $1 / V$ & r & صبحى كاملاً \\
\hline $1 \cdots$ & $1 T$. & جمع كل \\
\hline
\end{tabular}

براى بررسى ارتباط كيفيت زندكى و ابعاد آن با تيبهـاى

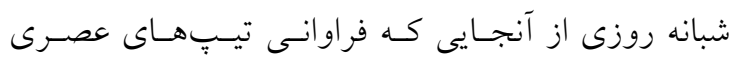

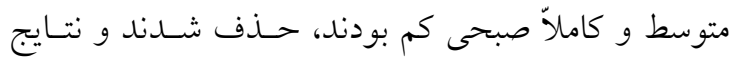

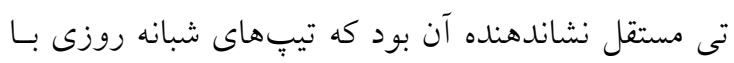


جدول شماره 0: شاخصهاى عددى ابعاد كيفيت زندكى در افراد داراى اختلال بيمارى صرع مراجعه كننده به انجمن صرع شهر تهران بر

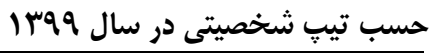

\begin{tabular}{|c|c|c|c|c|c|}
\hline \multirow[t]{2}{*}{ نتيجه آزمون تى مستقل } & \multicolumn{2}{|c|}{ متوسط صبحى } & \multicolumn{2}{|c|}{ بينابينى } & \multirow[t]{2}{*}{ كيفيت زندگى و ابعاد آن } \\
\hline & انحراف معيار & ميانگين & انحراف معيار & ميانكين & \\
\hline $\begin{array}{c}t=\cdot / \cdot r \wedge \quad d f=1 / r \\
P=\cdot / q V V\end{array}$ & $r_{N / O}$ & $07 / 90$ & rN/TA & OV/MA & نغر انى از تشنج \\
\hline $\begin{array}{c}t=1 / 107 \quad d f=1 / r \\
P=\cdot / r 0\end{array}$ & rT/O & $\varepsilon V / \cdot r$ & $19 / \mathrm{NV}$ & Or/TK & سلامت روحى \\
\hline $\begin{array}{c}t=1 / 7 r \mid \quad d f=1 / r \\
P=\cdot / 1 \cdot \wedge\end{array}$ & IV/NO & $\{1 / 7\}$ & $1 N / \varepsilon 7$ & $\sum V / 9 \Lambda$ & انرزى/ خستخى \\
\hline $\begin{array}{c}t=1 / 9 \mathrm{Tr} \quad \mathrm{df}=11 \mathrm{r} \\
\mathrm{P}=. / .0 \mathrm{~V}\end{array}$ & $r \cdot / r l$ & ro/.1 & ro/TV & $\{q / T\}$ & عملكرد شناختى \\
\hline $\begin{array}{c}\mathrm{t}=\cdot / 171 \quad \mathrm{df}=11 \mathrm{r} \\
\mathrm{P}=\cdot / \mathrm{NV} r\end{array}$ & $r / / \varepsilon V$ & $00 / \cdot V$ & ry/or & $07 / 79$ & اثرات دارويى \\
\hline $\begin{array}{c}\mathrm{t}=1 / \mathrm{r} \quad \mathrm{df}=11 \mathrm{r} \\
\mathrm{P}=\cdot / \pi \mu r\end{array}$ & $r V / \varepsilon V$ & 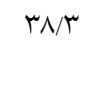 & rT/ru & $\varepsilon V / \cdot 9$ & عملكرد اجتماعى \\
\hline $\begin{array}{c}\mathrm{t}=\cdot / r \mathrm{C}_{0} \mathrm{df}=11 \mathrm{r} \\
\mathrm{P}=\cdot / \mathrm{Ar}\end{array}$ & $r \mu / A r$ & $\varepsilon \eta / \wedge q$ & $r \varepsilon / r q$ & EO/NT & كيفيت زندگى كلى \\
\hline $\begin{array}{c}t=1 / 179 \quad \mathrm{df}=1 / r \\
P=\cdot / r \varepsilon 0\end{array}$ & $r I / T \varepsilon$ & $\varepsilon 0 / \varepsilon V$ & Tr/O\& & $01 / \pi \varepsilon$ & نمره كل كيفيت زندگى \\
\hline
\end{tabular}

جدول شماره ا: نتايج رترسيون خطى عوامل مرتبط با كيفيت زندَى در افراد داراى اختلال بيمارى صرع مراجعه كننده به انجمن صرع

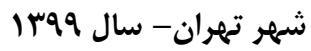

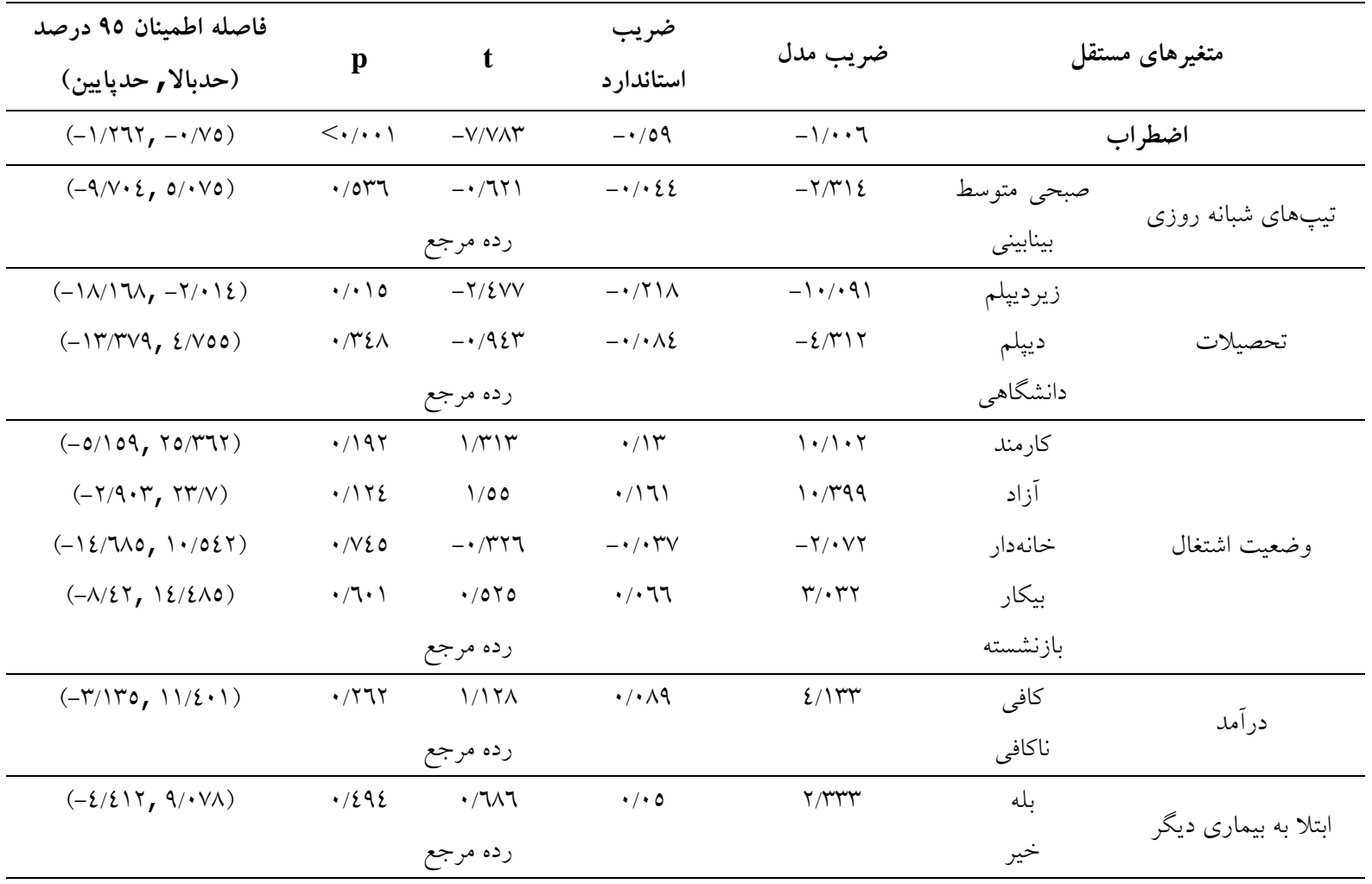


اضطر ابآور علائم و حملاتشان را كتترل نمايند. بنـابراين بحث و نتيجه كيرى

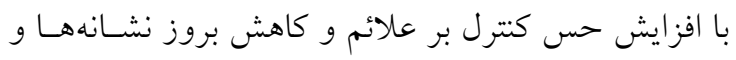

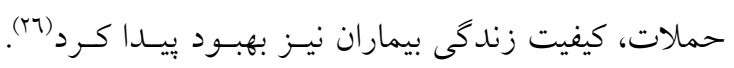

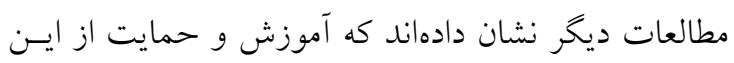

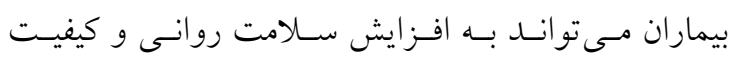

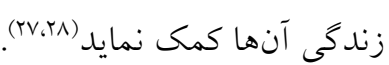

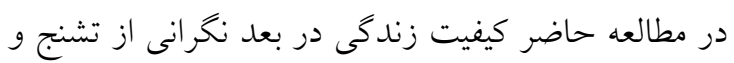

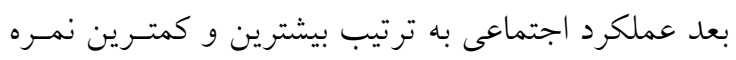

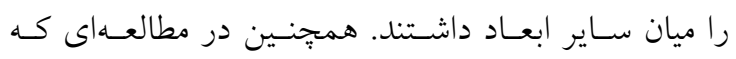

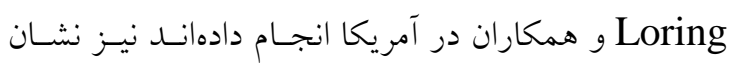

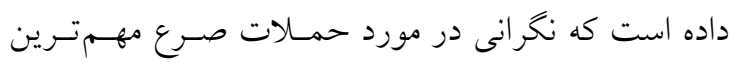

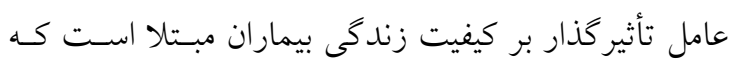

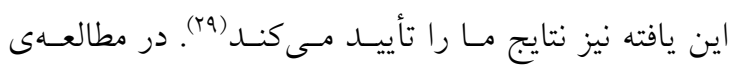

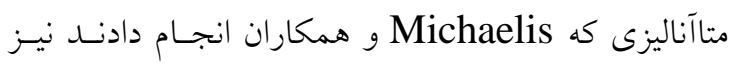
بيشترين نمره در ميان ابعاد كيفيت زندكى در بعد نحرانس

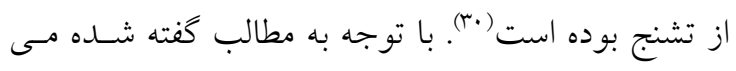

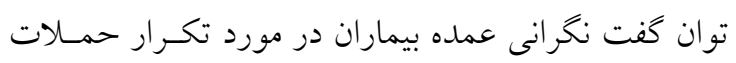

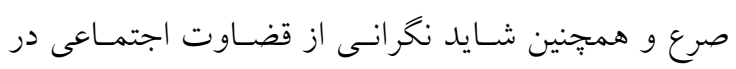

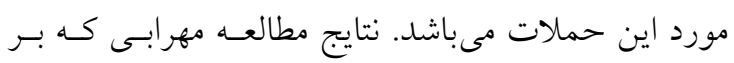

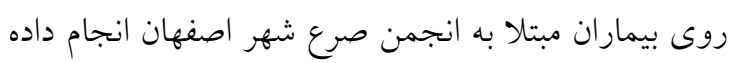

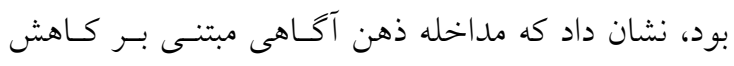
استرس به طور معنىدارى موجب بهبود مؤلفههـا و ابعـاد

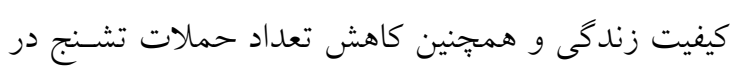

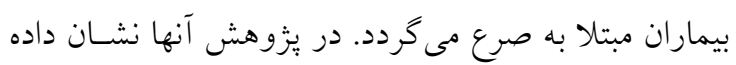

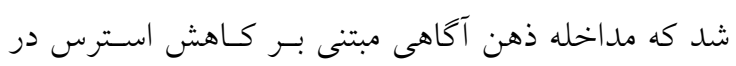
بهبود كيفيت زندكى و كاهش تعداد حمـلات تشــنج ايسن فئس

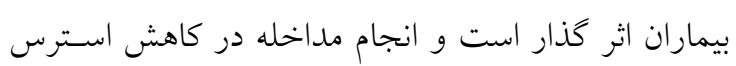

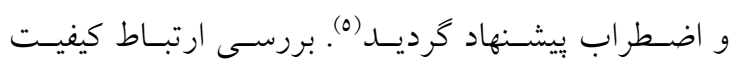

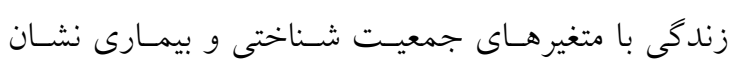

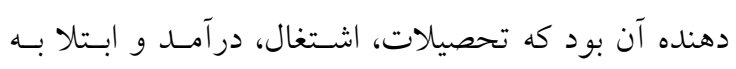

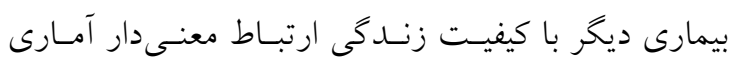
داشته است. از اين ميان اضطراب و تحصسيلات زيسردييلم

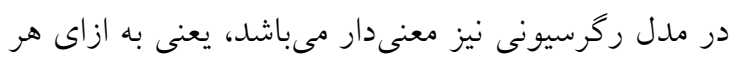

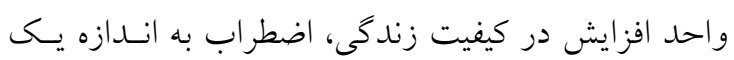

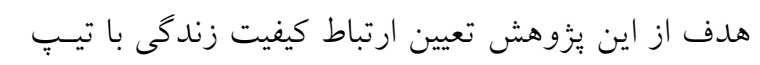

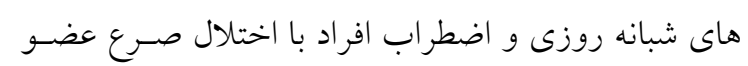

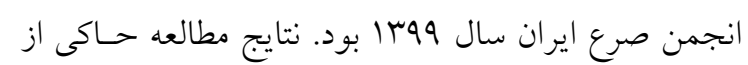

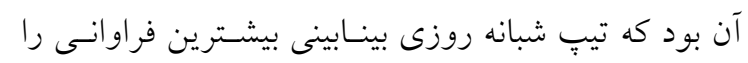

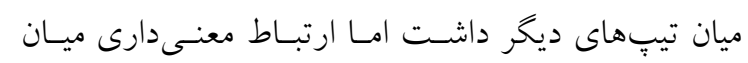

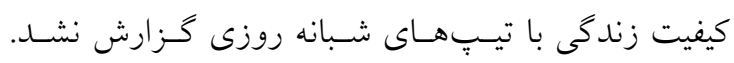

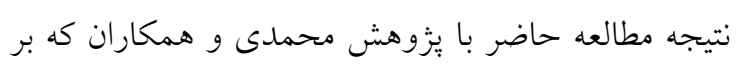

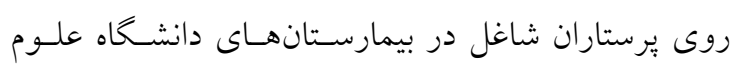

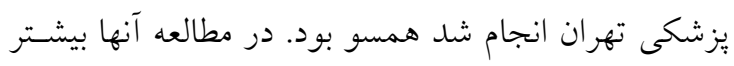
نمونها، تيب بينابينى بودند و هيج كونه ارتبـاط معنسى دار

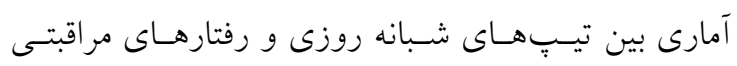

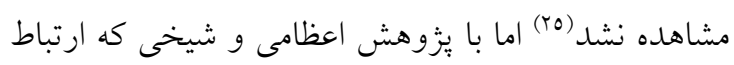

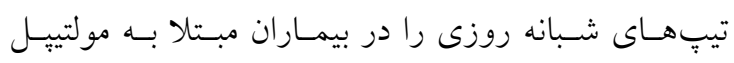

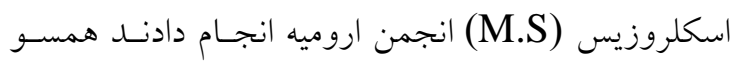

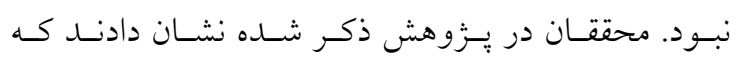

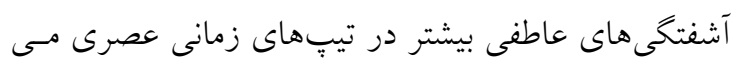

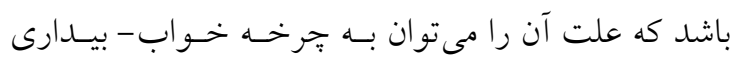

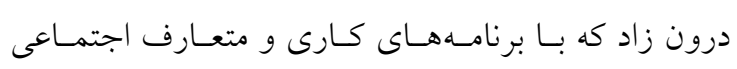

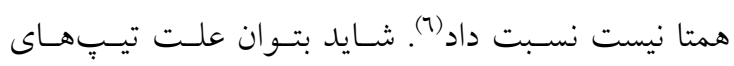

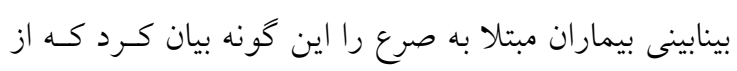

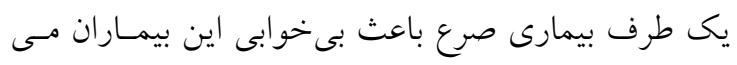

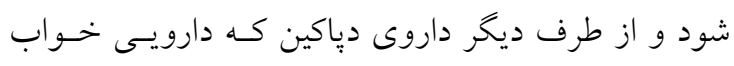

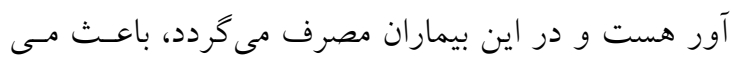
شود كه خواب آنها ريتم مشخصى نداشته باشد.

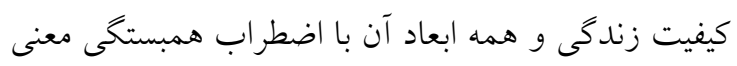

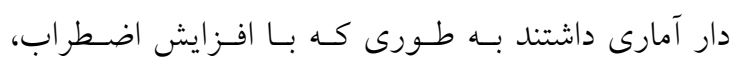

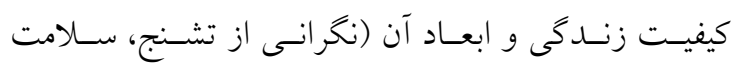

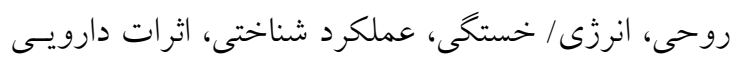

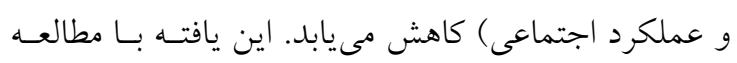

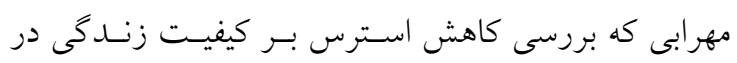

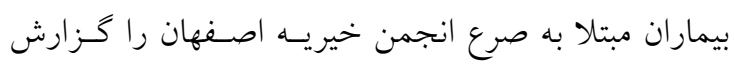

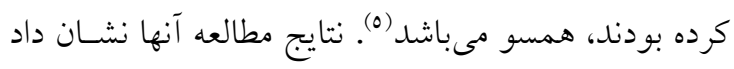

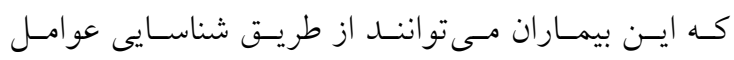


بين حملات رنج ببرند (TV). با توجه به اينكه اين مطالعه

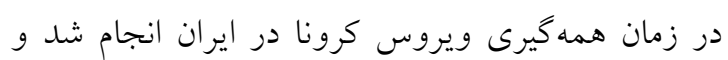

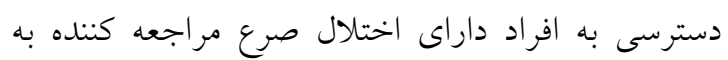

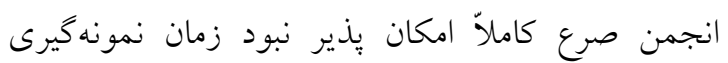
طولانى شد. در بيماران مبتلا به صرع بهبود كيفيت زنسـدى بيمـاران از جنبههاى مهم سلامتى به شمار مىرود. انجمن صرع بايـــ

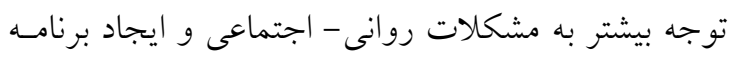

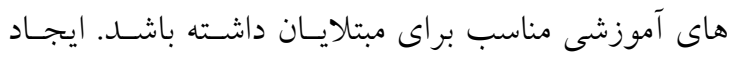

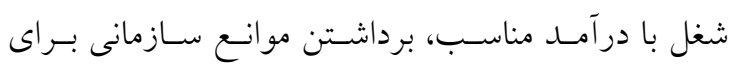

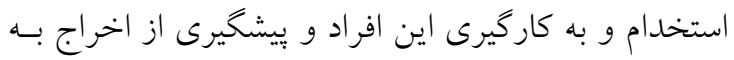

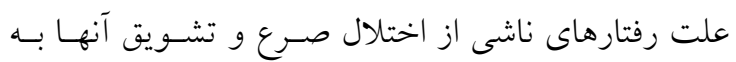

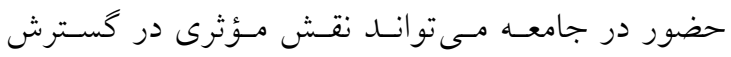

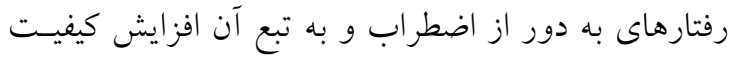

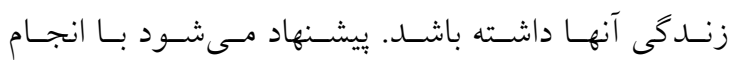

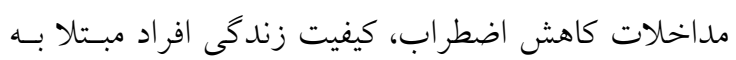

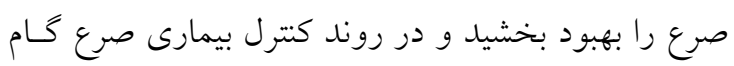

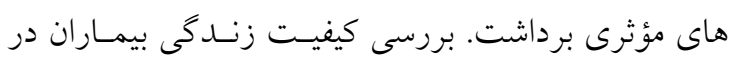

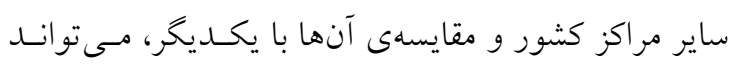

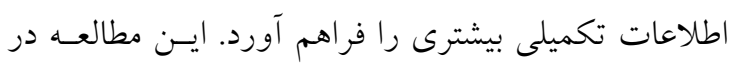

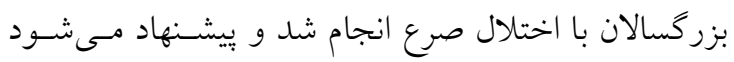
كيفيت زندكى در كودكان و نوجوانان داراى اختلال صرع نيز بررسى كردد. تعارض منافع: هيج گونه تعارض منافع توسط نويسندگان بيان نشده است.

\section{تقدير و تشكر}

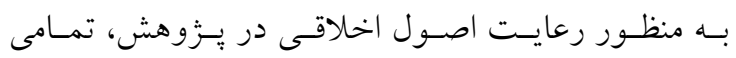

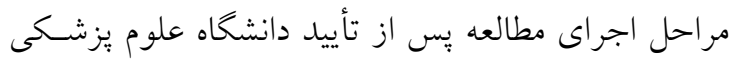

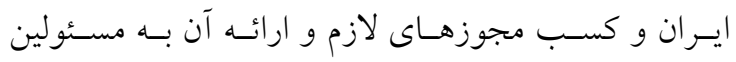

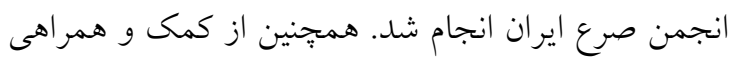

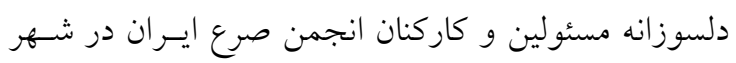

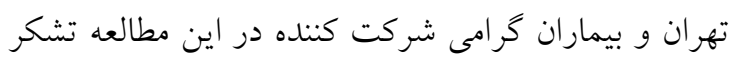

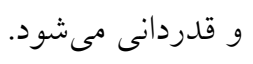

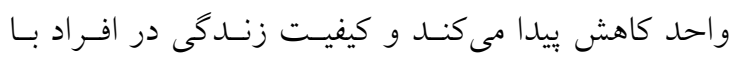

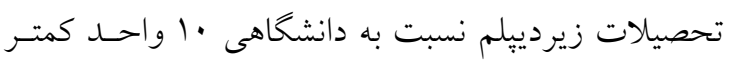

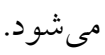

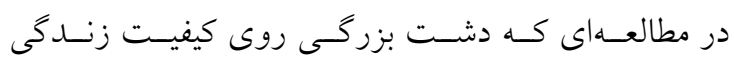

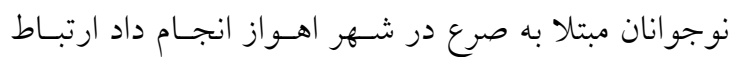

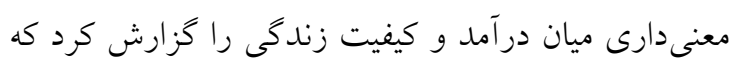

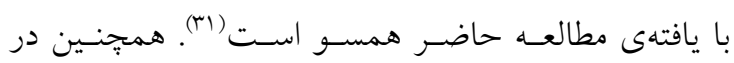

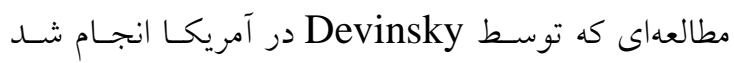

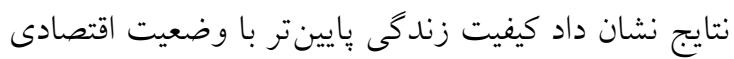

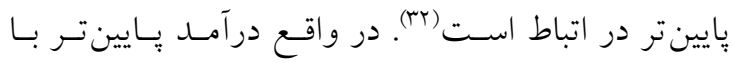

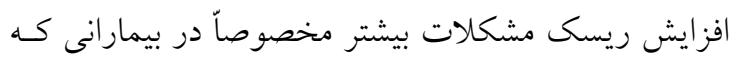
مبتلا به بيمارى هاى مزمنى هم:جون صـرع هستـند همـراه

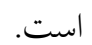

در مطالعه دارابى و همكاران كه به تعيين عوامل جمعيتسى

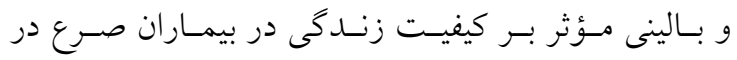
بيمارستان سينا تهران انجام شد، نتايج نشان دادئ دادند كه سال

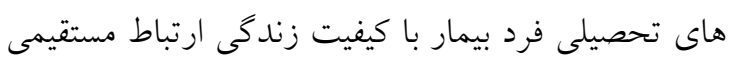

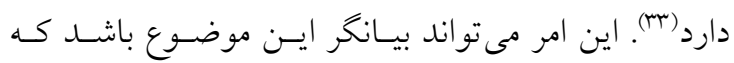

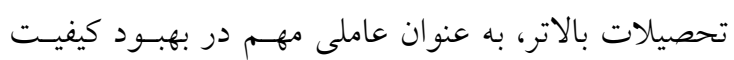
زندكى اين بيماران است. نتايج مطالعه سادات و همكاران

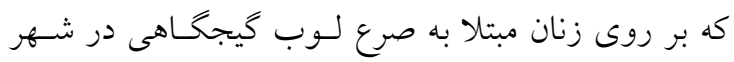

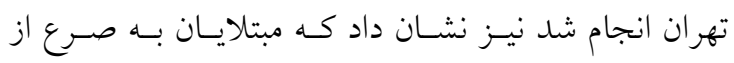

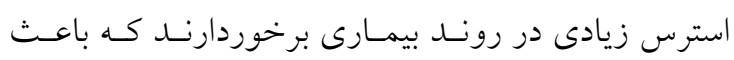

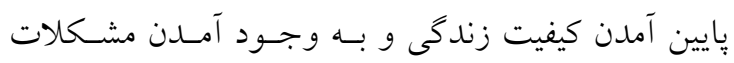

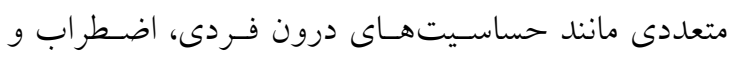

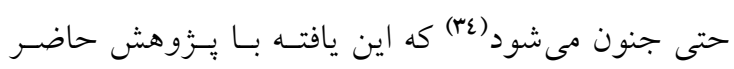
همسو مى باشد. نتايج يزوهش حاضر نشان داد بيشتر شركت كنندكان (T/0 درصد) نمره بالايى از نظر وضعيت اضطراب (بهنجار) داشتند كه همسو با مطالعه انجام شده توسط Yeni نتايج مطالعه كاظمى فروز و همكاران نشان داد كه لازم

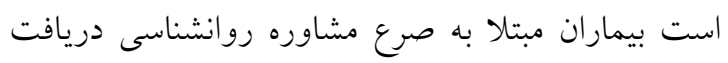
نمايند تا به ميزان كمترى از اختلالات روانشناختى در فاز 


\section{References}

1. Ablah E, Hesdorffer DC, Liu Y, Paschal AM, Hawley S, Thurman D, Hauser WA, Prevalence of Epilepsy in Rural Kansas Study Group. Prevalence of epilepsy in rural Kansas. Epilepsy research. 2014;108(4):792-801.

2. Haji A, Mahmoud Fakhe H. Relationship of social support and self-management with quality of life in patients with epilepsy. Journal of Health and Care. 2015;17(3):240-7. [Persian]

3. Neligan A, Sander JW. The incidence and prevalence of epilepsy. London: UCL Institute of Neurology. 2009.

4. Panagariya A, Sharma B, Dubey P, Satija V, Rathore M. Prevalence, demographic profile, and psychological aspects of epilepsy in North-Western India: a community-based observational study. Annals of neurosciences. 2018;25(4):177-86.

5. Mehrabi F. The effectiveness of mindfulness intervention based on stress reduction on quality of life and the number of seizure attacks in patients with epilepsy. Shenakht journal of psychology \& psychiatry. 2019;6(1):152-9. [Persian]

6. Azamy SH. Evaluation of Quality of Life and Its Relationship with Boarding Ranges in Patients with Multiple Sclerosis (Ms) Ms Community in Urmia. Nursing And Midwifery Journal. 2017;15(8):575-83. [Persian]

7. Kowalski C, Ommen O, Driller E, Ernstmann N, Wirtz MA, Köhler T, Pfaff H. Burnout in nurses-the relationship between social capital in hospitals and emotional exhaustion. Journal of Clinical nursing. 2010;19(11-12):1654-63.

8. Nicoletti F, Bruno V, Ngomba RT, Gradini R, Battaglia G. Metabotropic glutamate receptors as drug targets: what's new?. Current Opin Pharmacol. 2015;20:89-94.

9. Maganti RK, Jones MV. Untangling a Web: Basic Mechanisms of the Complex Interactions Between Sleep, Circadian Rhythms, and Epilepsy. Epilepsy Currents. 2021;21(2):105-10.

10. Piperidou C, Karlovasitou A, Triantafyllou N, Terzoudi A, Constantinidis T, Vadikolias K, Heliopoulos I, Vassilopoulos D, Balogiannis S. Influence of sleep disturbance on quality of life of patients with epilepsy. Seizure. 2008;17(7):588-94.

11. Yazdi Z, Sadeghniiat-Haghighi K, Naimian S, Zohal MA, Ghaniri M. Prevalence of sleep disorders and their effects on sleep quality in epileptic patients. Basic and clinical neuroscience. 2013;4(1):36.

12. Zahiroddin AR, Ghoreishi F. A survey of depressive symptoms in patients. Research in medicine. 2006;30(4):305-8. [Persian]

13. Maroufi A, Khomand P, Ahmadiani S, Alizadeh NS, Gharibi F. Prevalence and quality of anxiety in patients with epilepsy. Epilepsy \& Behavior. 2014;32:34-7. [Persian]

14. Dehghanifiroozabadi M, Manshaee G, Danae Sij Z, Sharifzadeh G. Effectiveness of cognitive behavioral stress management on depression and anxiety symptoms of patients with epilepsy and migraine. Journal of Birjand University of Medical Sciences. 2015;21(4):407-15. [Persian]

15. Kwon OY, Park SP. Depression and anxiety in people with epilepsy. J Clin Neurol. 2014;10(3):175-88.

16. Baker DA, Caswell HL, Eccles FJ. Self-compassion and depression, anxiety, and resilience in adults with epilepsy. Epilepsy \& Behavior. 2019;90:154-61.

17. Gilliam FG. Diagnosis and treatment of mood disorders in persons with epilepsy. Current Opin Neurol. 2005;18(2):129-33.

18. Jones JE, Hermann BP, Woodard JL, Barry JJ, Gilliam F, Kanner AM, Meador KJ. Screening for major depression in epilepsy with common self-report depression inventories. Epilepsia. 2005;46(5):731-5.

19. Nouhi S, Azar M, Karamad A, Shafiei Kandjani AR, Amiri M, Habibi M, Asari.S. Frequency of Symptoms of Depression, General Anxiety, Obsessive- Compulsive and Phobia in Interictal Phase in Patients with Epilepsy. Medical Sciences Journal of Islamic Azad University. 2008;18(1):39-4. [Persian]

20. Roshannia S, Rezaei F, Ghadampoor E. An Overview of Psychological Factors in Epilepsy. The Neuroscience Journal of Shefaye Khatam. 2017;5(2):110-23. [Persian]

21. Seyedoshohadaee M, Mohammadi H, Seyedfatemi N, Mehran A. Relationship between the circadian types and nurses' caringbehaviours. Koomesh. 2015;16(3):389-96. [Persian] 
22. Kaviani H, Mousavi AS. Psychometric properties of the Persian version of Beck Anxiety Inventory (BAI). Tehran University Medical Journal. 2008;66(2):136-40.

23. Beck AT, Epstein N, Brown G, Steer RA. An inventory for measuring clinical anxiety: psychometric properties. J consul clin psychol. 1988;56(6):893.

24. Montazeri A, Togha M, Vahdaninia MA, Ebrahimi F. Psychometric properties of the Iranian version of Quality of Life in Epilepsy Inventory 31-Item. Payesh 2010;9(4):417-24. [Persian]

25. Mohammadi H, Seyedshohadai M, Seyedfatemi N, Mehran A. Relationship between circadian types and nurses' caring behaviors. Iran Journal of Nursing. 2014;27(90):44-53. [Persian]

26. O'Neill CA. Neurobiological, demographic, and psychosocial factors associated with depression and anxiety in individuals with epilepsy. University of Hartford; 2005.

27. Kazemiforouz F, Shirvani S, Khomand P, Marofi A, Araste M, Gharibi F, Abdolmalaki M, Rajabi M. Prevalence of mood-behavioral disorders (depression, anxiety, obsessive-compulsive Disorder, phobia) in epileptic patients in Sanandaj in2015. Zanko Journal of Medical Sciences. 2017;18(57):11-9. [Persian]

28. Yeni K, Tulek Z, Simsek OF, Bebek N. Relationships between knowledge, attitudes, stigma, anxiety and depression, and quality of life in epilepsy: A structural equation modeling. Epilepsy \& Behavior. 2018;85:212-7.

29. Loring DW, Meador KJ, Lee GP. Determinants of quality of life in epilepsy. Epilepsy \& Behavior. 2004;5(6):976-80.

30. Michaelis R, Tang V, Nevitt SJ, Wagner JL, Modi AC, LaFrance Jr WC, Goldstein LH, Gandy M, Bresnahan R, Valente K, Donald KA. Psychological treatments for people with epilepsy. Cochrane Database of Systematic Reviews. 2020(8).

31. Dasht Bozorgi B, Majdinasab N, Sabeti Z, Alijani RH, Latifi SM. The effect of progressive muscular relaxation on quality of life in adolescents with epilepsy. Jundishapur Scientific Medical Journal. 2010;9(5):465-72. [Persian]

32. Devinsky O, Westbrook L, Cramer J, Glassman M, Perrine K, Camfield C. Risk factors for poor health-related quality of life in adolescents with epilepsy. Epilepsia. 1999;40(12):1715-20.

33. Darabi L, Montazeri A, Ebrahimi F, Vahdaninia M, Togha M. Clinical and demographic characteristics and quality of life in patients with epilepsy. Payesh (Health Monitor). 2010;9(2):215-21. [Persian]

34. Sadat S, Tangestani Y. The effectiveness of mindfulness training on psychological health in patients with temporal lobe epilepsy. J Nov. Appl Sci. 2015;4(9):923-27. 